\title{
Effects of Using Dynamic Office Chairs on Posture and EMG in Standardized Office Tasks
}

\author{
Rolf Ellegast ${ }^{1}$, Rene Hamburger ${ }^{1}$, Kathrin Keller ${ }^{1}$, Frank Krause ${ }^{2}$, \\ Liesbeth Groenesteijn ${ }^{2}$, Peter Vink ${ }^{2}$, and Helmut Berger ${ }^{3}$ \\ ${ }^{1}$ BG Institute for Occupational Safety (BGIA), Alte Heerstrasse 111, 53757 Sankt Augustin, \\ Germany \\ ${ }^{2}$ TNO Work and Employment, Polarisavenue 151, 2130 AS Hoofddorp, The Netherlands \\ ${ }^{3}$ BG for Administration, Nikolaus Dürkopp-Str. 8, 33602 Bielefeld, Germany
}

\begin{abstract}
In the paper a measuring system for the comparative posture and EMG analysis of office chairs is presented. With the system four specific dynamic office chairs that promote dynamic sitting and therefore aim to prevent musculoskeletal disorders (MSD), were analyzed in comparison to a reference chair in two different standardized tasks (intensive mouse use and sorting files). Exemplary results of the ongoing study suggest that postures and the electrical activities of the erector spinae and trapezius muscles depend more on the tasks performed than on the use of a particular type of office chair. This still has to be proved by statistical analysis.
\end{abstract}

Keywords: office chair, EMG, posture, measuring device, dynamic sitting.

\section{Introduction}

Working frequently and continuously in static sitting postures at visual display unit (VDU) workplaces can cause muscular tension and musculoskeletal disorders [1]. Static muscular workloads particularly of the shoulder and neck can occur as well as functional underuse of certain muscle groups, such as the back and abdominal muscles [2]. In the development of office chairs, the concept of dynamic sitting has therefore been encouraged in recent years. By introducing structural elements that give the seat pan a dynamic mounting or active rotation of its own, some manufacturers of office chairs have created special chair characteristics that promote dynamic sitting with the aim of preventing musculoskeletal disorders at office and monitor workstations.

Various measurement methods for the quantification of the musculoskeletal load situation during seated activities at office workplaces and VDTs have been described in the literature. One example of this is stadiometry, in which the decrease in body height over the course of a day is adopted as the parameter for spinal loading [3], [4]. Other methods include electromyography (EMG), in which the muscle activity, e.g. of the back muscles, is taken as a measure of muscular stressing [5], or the measurement of body posture and movement as a means of quantifying spinal postures in particular [6], [7]. 
In the context of the study presented here, a measuring system has been created that combines posture and EMG measuring methods in such a way that the load situation during seated office work can be ergonomically analysed in both laboratory and real-life environments. Comparative posture and EMG measurements were conducted for four dynamic office chairs and one conventional reference office chair in two standardized office tasks (intensive mouse use and sorting files). Herewith the effects on posture and EMG depending on office task type and chair characteristics are analysed.

\section{Methods}

The study was carried out at a realistic VDU office workplace in laboratory. 10 test subjects ( 5 men and 5 women) took part in the laboratory tests. The mean ages were 35.4 years (SD 12.1 years) for the men and 34.8 (SD 12.7) for the women. Body heights ranged from 1.75 to $1.86 \mathrm{~m}$ for the men and from 1.62 to $1.68 \mathrm{~m}$ for the women. Body weights varied from 76 to $100 \mathrm{~kg}$ for the men and from 47 to $78 \mathrm{~kg}$ for the women. Each subject tested a total of five office chairs (4 particularly dynamic ones labelled A, B, C and E and 1 reference office chair - labelled D) during the performance of the following standardized tasks (duration of activity in brackets):

- Intensive use of the mouse (20 minutes)

- Sorting files (10 minutes)

Body postures and movements were measured with the CUELA system [8]. This person-centered measuring system consists of movement sensors (3D accelerometers and gyroscopes) as well as a miniature data storage unit with a flash memory card, which can be attached to the subject's clothing [9], [10]. In this study a special version of the system adopted for sitting postures was used [11] and the sensors were attached to the subject's skin.

From the measured signals, the following body/joint angles were calculated: Head inclination (sagittal and lateral), flexion/extension and lateral flexion of the spine in the thoracic (Th3) and lumbar spinal regions (L1 and transition to L5/S1), trunk inclination and the spatial position of the upper and lower legs (right and left).

From the kinematic measurements at the thoracic spine Th3 and lumbar spine L5/S1 physical acitivity intensities (PAI) were determined by calculating a sliding standard deviation of the high-passed filtered vector magnitude of the 3D acceleration signals (time window: $1 \mathrm{~s}$ ).

Surface electromyography (EMG) was used for measuring the muscle activity of the trapezius muscle (right/left) and erector spinae muscle (right/left) with the CUELA EMG signal processor for long-term analysis [12], [13]. To assess the EMG signals, the root mean square values (RMS values) were calculated from the raw EMG data over consecutive time windows ( $0.3 \mathrm{~s})$. To normalize the RMS values, reference activities are performed at the beginning of all measurement so that all muscle activities stand in relation to a reference voluntary contraction (\% RVC). The $100 \%$ RVC values for each muscle were defined as the 50th Percentile RMS values of 


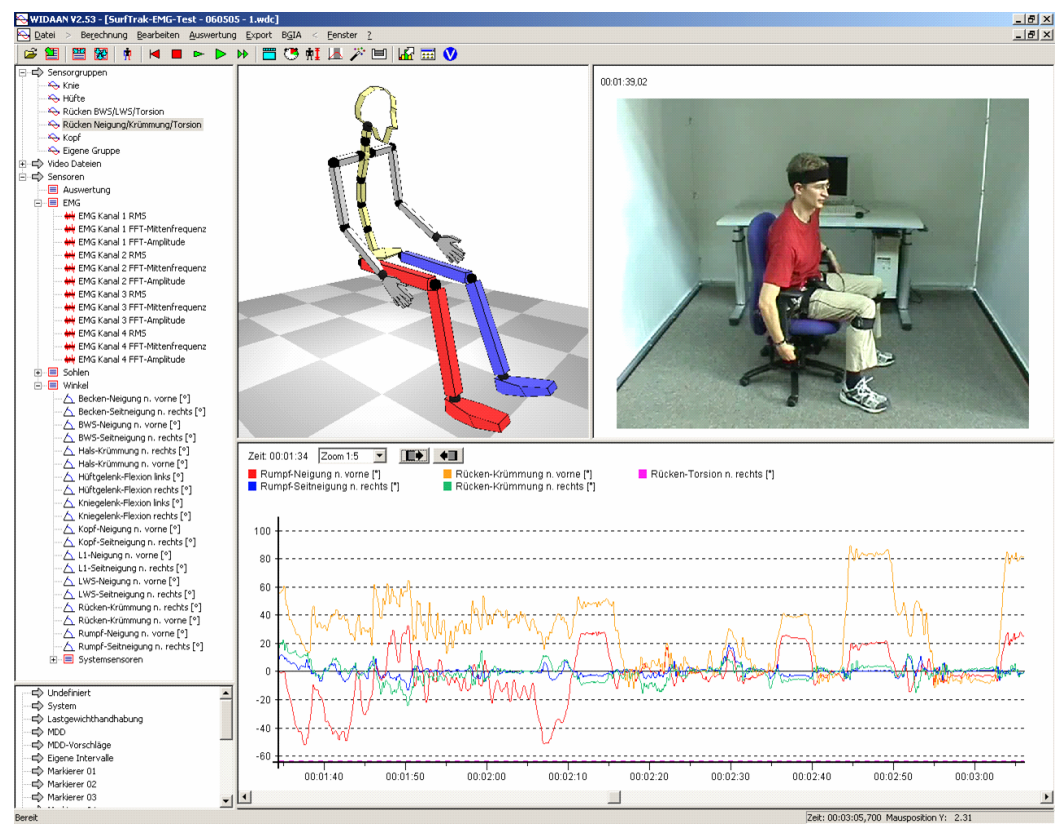

Fig. 1. Data representation with the CUELA software by video (right, above), animated figure (left, above) and selected data time graph (below)

a calibration interval at the beginning of each measurement (duration: $3 \mathrm{~s}$ ), where the subjects were standing with their arms abducted at $90^{\circ}$ holding a weight of $5 \mathrm{~kg}$ (females) or $10 \mathrm{~kg}$ (males). The $0 \% \mathrm{RVC}$ values for each muscle were set as the minimum values of another calibration interval at the beginning of each measurement (duration $3 \mathrm{~s}$ ), where the subjects were standing upright in a neutral relaxed posture.

All the measured data were synchronously recorded at a sampling rate of $50 \mathrm{~Hz}$ in a data logger of the CUELA measuring system. They can be depicted with the CUELA software together with the digitalized video recording of the workplace situation and a 3D animated figure (Figure 1). For statistical evaluation the software includes a calculation of the characteristic values of the frequency distributions (5th, 25th, 50th, 75th and 95th percentile) of each joint/body angle, physical activity intensity (PAI) and EMG recording. The frequency distributions of each measurement can be depicted in a box-plot diagram. From the box-plot diagrams for each subject, task and chair type the mean values for all subjects of the characteristic frequency distributions (5th, 25th, 50th, 75th and 95th percentile) were calculated and displayed in box-plot diagrams (mean for ten subjects, see e. g. Figure 2).

\section{Results}

In the following exemplary results of the ongoing study are presented. The frequency distributions are depicted as box-plot diagrams. The boundaries of the box are defined by the 25 th and 75 th percentile of the measured frequency distribution. The median (50th 
percentile value) of the distribution is given as the central value in the box. The edge values of the distributions (5th and 95th percentile values) are marked with whiskers.

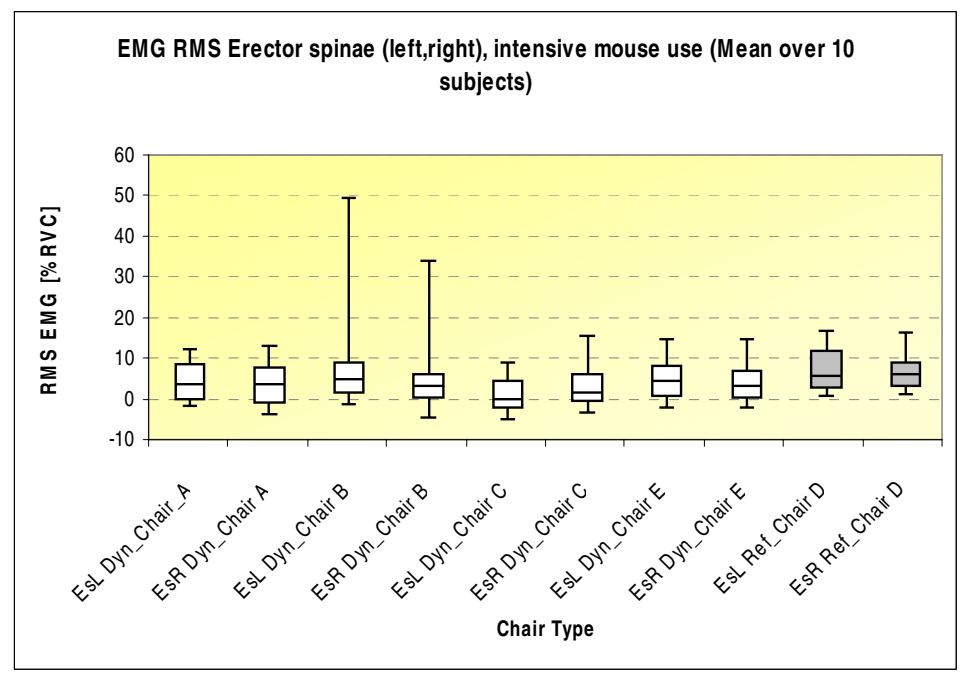

Fig. 2. Box-plot diagram of the mean values (over 10 subjects) of the 5th, 25th, 50th, 75th and 95th percentiles of the EMG RMS values (in \% RVC) for the erector spinae muscle left (EsL) and right (EsR) for all dynamic chairs (Dyn_Chair A, B, C, E) and the reference chair (Ref_Chair D) for the task "intensive mouse use"

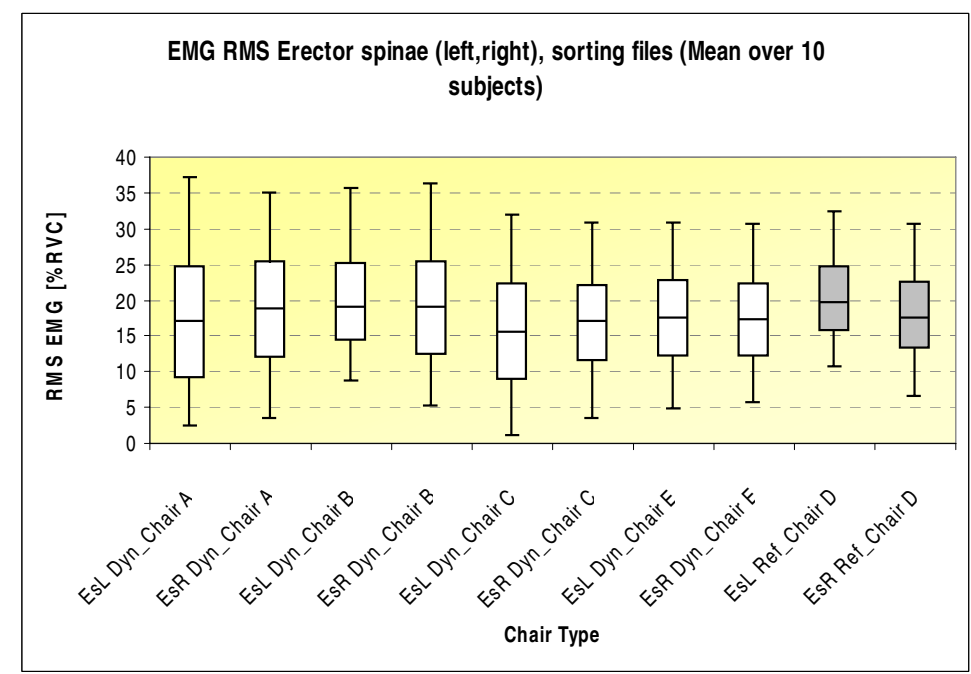

Fig. 3. Box-plot diagram of the mean values (over 10 subjects) of the 5th, 25th, 50th, 75 th and 95th percentiles of the EMG RMS values (in \% RVC) for the erector spinae muscle left (EsL) and right (EsR) for all dynamic chairs (Dyn_Chair A, B, C, E) and the reference chair (Ref_Chair D) for the task "sorting files" 
Figure 2 and Figure 3 show the mean values (over 10 subjects) of the 5th, 25th, 50th, 75th and 95th percentiles of the EMG RMS values (in \% RVC) for the erector spinae muscles left and right for all chairs in the tasks "intensive mouse use" (Figure 2) and "sorting files" (Figure 3). The RMS values of the erector spinae muscle are not high for both office tasks and all chairs. As expected for the more dynamic task of sorting files the RMS values for the erector spinae muscle are generally higher for all chairs ( $>15 \%$ RVC at the 50th percentile for all chairs and $>20 \%$ RVC at the 75th percentile). There is not much difference between the frequency distributions of the dynamic chairs in comparison to the refernce chair (exception: dynamic chair B for the task "intensive mouse use").

Figure 4 and Figure 5 show the mean values (over 10 subjects) of the 5th, 25th, 50th, 75th and 95th percentiles of the EMG RMS values (in \% RVC) for the trapezius muscles left and right for all chairs in the tasks "intensive mouse use" (Figure 4) and "sorting files" (Figure 5). The RMS values of the trapezius muscle are much dependant on the task performed. The static mouse task yield to low RMS values of less than 5\% RVC for the 50th percentile $(<7 \% \mathrm{RVC}$ for the 75 th percentile), whereas for the dynamic task of sorting files high RMS values of up to $50 \%$ RVC (50th percentile) and 65\% RVC (75th percentile) were measured. A comparison of the frequency distributions of the dynamic chairs and the reference chair shows differences for the dynamic chair $\mathrm{B}$ in the static mouse task and for the dynamic chairs A, B and C (trapezius muscle right) for the sorting file task.

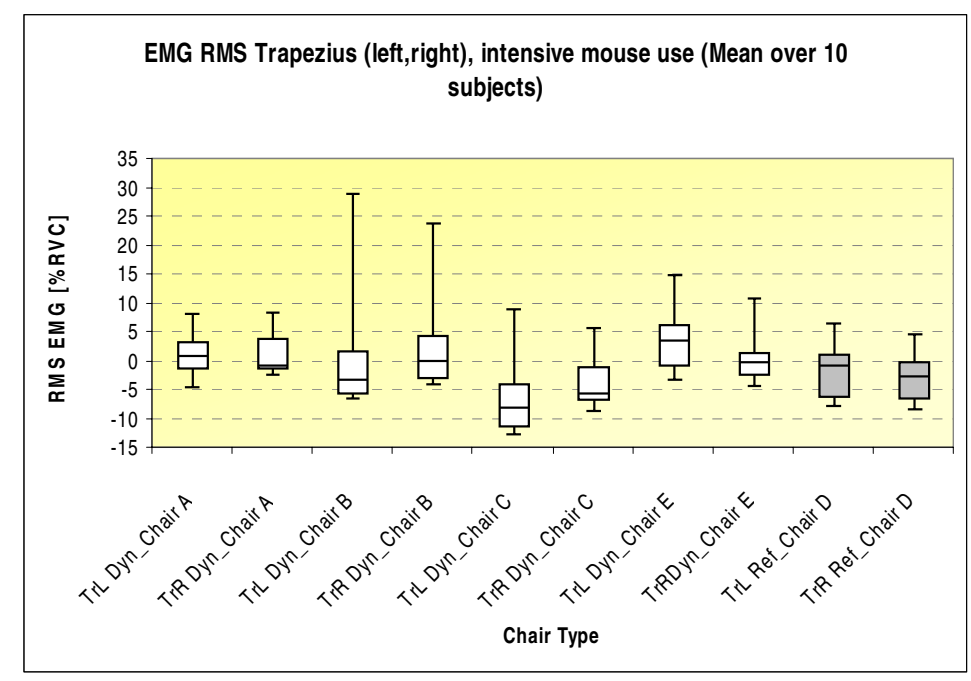

Fig. 4. Box-plot diagram of the mean values (over 10 subjects) of the 5th, 25th, 50th, 75th and 95th percentiles of the EMG RMS values (in \% RVC) for the trapezius muscle left (TrL) and right (TrR) for all dynamic chairs (Dyn_Chair A, B, C, E) and the reference chair (Ref_Chair D) for the task "intensive mouse use" 


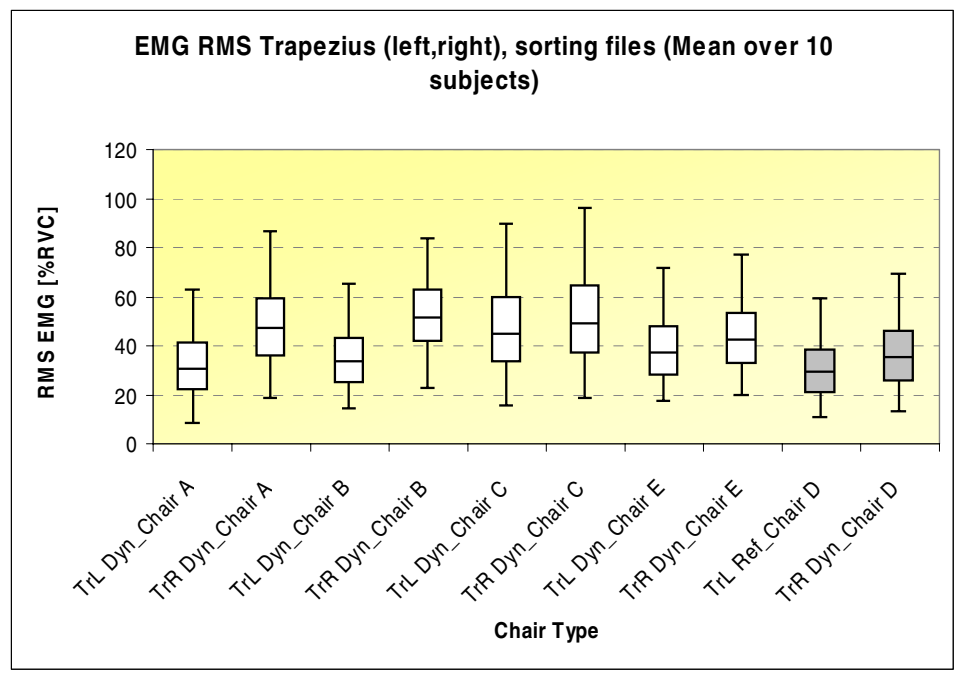

Fig. 5. Box-plot diagram of the mean values (over 10 subjects) of the 5th, 25th, 50th, 75th and 95th percentiles of the EMG RMS values (in \% RVC) for the trapezius muscle left (TrL) and right (TrR) for all dynamic chairs (Dyn_Chair A, B, C, E) and the reference chair (Ref_Chair D) for the task "sorting files"

Figure 6 and Figure 7 show the mean values (over 10 subjects) of the 5th, 25th, 50th, 75th and 95th percentiles of the calculated physical activity intensity (PAI) (in $\% \mathrm{~g}$ ) for the thracic spine at Th 3 for all chairs in the tasks "intensive mouse use" (Figure 6) and "sorting files" (Figure 7). For the mouse task all PAI values are very low $(<0.21 \% \mathrm{~g}$ for the 75 th percentile and $<0.15 \% \mathrm{~g}$ for the 50th percentile). For the task of sorting files the PAI values are higher $(>0.6 \% \mathrm{~g}$ for the 75 th percentile and $>$ $0.4 \% \mathrm{~g}$ for the 50th percentile). A comparison of the box-plot diagrams of the dynamic chairs and the reference chair show not many differences for both tasks.

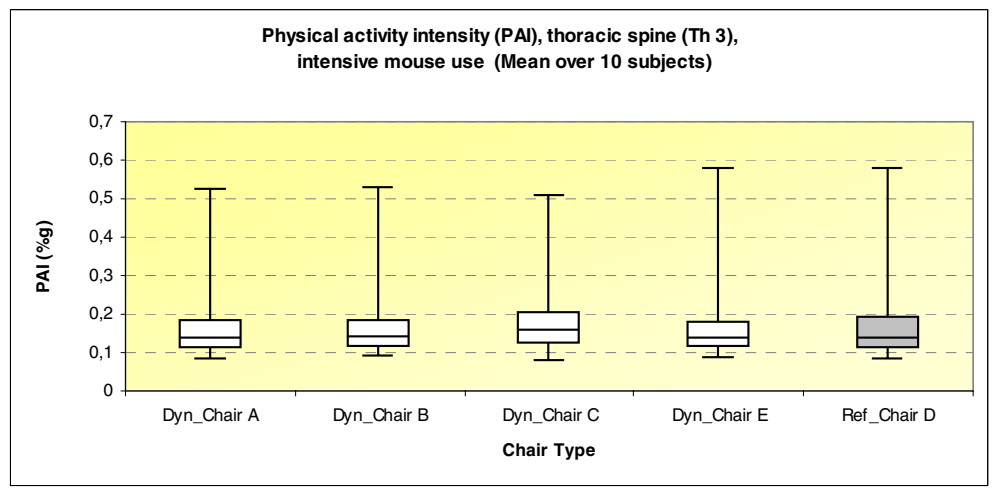

Fig. 6. Box-plot diagram of the mean values (over 10 subjects) of the 5th, 25th, 50th, 75th and 95th percentiles of the physical activity intensity (PAI) in \%g (\%acceleration of gravity) for all dynamic chairs (Dyn_Chair A, B, C, E) and the reference chair (Ref_Chair D) for the task "intensive mouse use" 


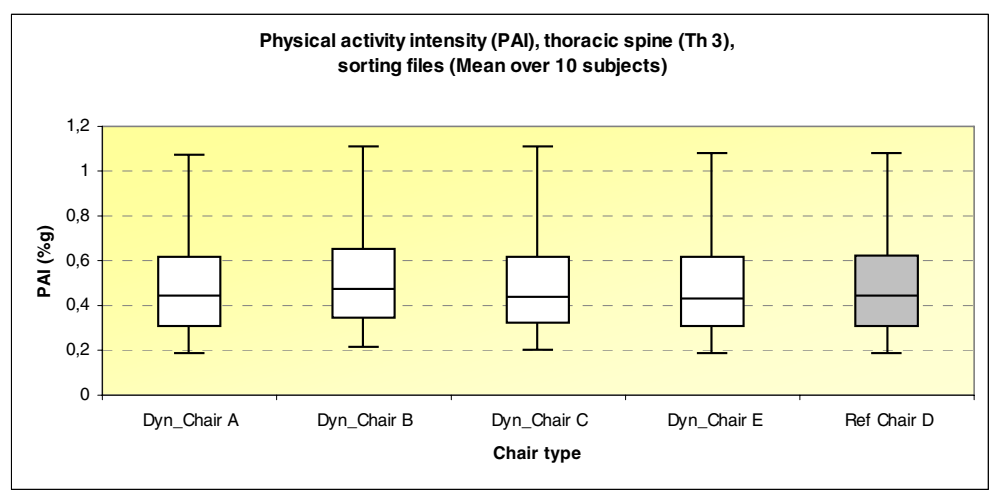

Fig. 7. Box-plot diagram of the mean values (over 10 subjects) of the 5th, 25th, 50th, 75th and 95th percentiles of the physical activity intensity (PAI) ) in \%g (\%acceleration of gravity) for all dynamic chairs (Dyn_Chair A, B, C, E) and the reference chair (Ref_Chair D) for the task "sorting files"

Figure 8 and Figure 9 show the mean values (over 10 subjects) of the 5th, 25th, 50th, 75th and 95th percentiles of the trunk inclination angles (in ${ }^{\circ}$ ) for all chairs in the tasks "intensive mouse use" (Figure 8) and "sorting files" (Figure 9). The mouse task was performed with the trunk mainly inclined backwards. The trunk inclination angles range from $-2^{\circ}$ to $-6^{\circ}$ for the 50th percentile. The sorting file task was performed with the trunk inclined forward most of the time. Here trunk inclination angles range from less than $2^{\circ}$ to more than $5^{\circ}$ for the 50th percentile. For both different tasks and all chairs the differences of the trunk inclination angles at the 95th percentile and the 5 th percentile are less than $15^{\circ}$. There is not much difference between the frequency distributions of the dynamic chairs and the reference chair.

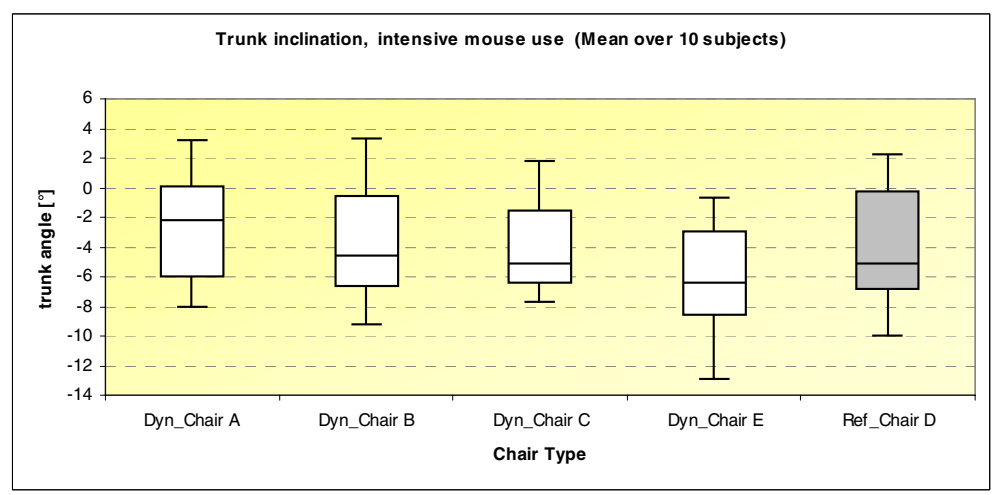

Fig. 8. Box-plot diagram of the mean values (over 10 subjects) of the 5 th, 25th, 50th, 75 th and 95th percentiles of the trunk inclination angle in $\left(^{\circ}\right)$ for all dynamic chairs (Dyn_Chair A, B, C, E) and the reference chair (Ref_Chair D) for the task "intensive mouse use" 


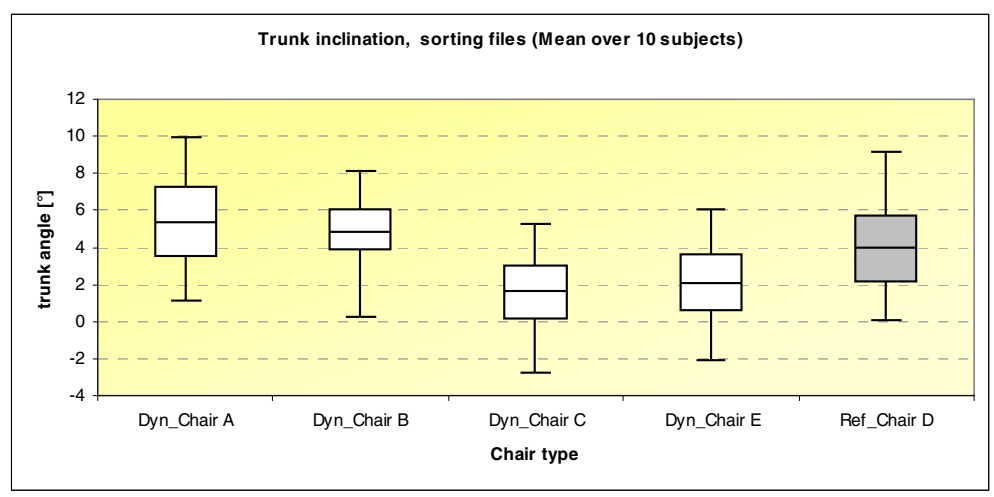

Fig. 9. Box-plot diagram of the mean values (over 10 subjects) of the 5th, 25th, 50th, 75th and 95th percentiles of the trunk inclination angle in $\left(^{\circ}\right)$ for all dynamic chairs (Dyn_Chair A, B, C, E) and the reference chair (Ref_Chair D) for the task "sorting files"

\section{Conclusions}

The measuring system developed permits the synchronous capture of different musculoskeletal load parameters. With the aid of the CUELA software, it is then possible to obtain, among other things, a very good general impression of muscle activity, body postures and movement for all test subject in relation to standardized tasks and different chair types. An initial evaluation of the ongoing study suggests that the load situation depends more on the tasks performed than on the use of a particular type of office chair. This still has to be proved by statistical analysis.

\section{References}

1. Rohlmann, A., Wilke, H.-J., Graichen, F., Bergmann, G.: Wirbelsäulenbelastung beim Sitzen auf einem Bürostuhl mit nach hinten kippbarer Rückenlehne. Biomed Techn (Berl) 47, 91-96 (2002)

2. Diebschlag, W., Heidinger, F.: Ergonomische Sitzgestaltung zur Prävention sitzhaltungsbedingter Wirbelsäulenschädigungen. Arbeitsmed Sozialmed Präventivmed (ASP) 25, 123-126 (1990)

3. Eklund, J.A.E., Corlett, E.N.: Shrinkage as a measure of the effect of load on the spine. Spine 9, 189-194 (1984)

4. Althoff, I., Brinckmann, P., Frobin, W., Sandover, J., Burton, K. (ed.): Die Bestimmung der Belastung der Wirbelsäule mit Hilfe einer Präzisionsmessung der Körpergröße. Schriftenreihe der Bundesanstalt für Arbeitsschutz (BAuA) (1993)

5. Bennet, D.L., Gillis, D.K., Portney, L.G., Romanow, M., Sanchez, A.S.: Comparison of integrated electromyographic activity and lumbar curvature during standing and during sitting in three chairs. Phys. ther 69, 902-913 (1989)

6. Adams, M., Hutton, W.C.: An electronic inclinometer technique for measuring lumbar curvature. Clin. Biomech (Bristol, Avon) 1, 130-134 (1986) 
7. Black, K.M., McClure, P., Polansky, M.: The influence of different sitting positions on cervical and lumbar posture. Spine 21, 65-70 (1996)

8. Ellegast, R.P., Kupfer, J.: Portable posture and motion measuring system for use in ergonomic field analysis. In: Landau, K. (ed.) Ergonomic software tools in product and workplace design, pp. 47-54 ,Verlag ERGON GmbH, Stuttgart (2000)

9. Glitsch, U., Ottersbach, H.J., Ellegast, R.P., Schaub, K., Jäger, M.: Musculo-Skeletal Loads on Flight Attendants when Pushing and Pulling Trolleys aboard Aircraft. J Aerosp. Eng. 113(1), 57-62 (2004)

10. Ellegast, R.P., Lesser, W., Herda, C., Hoehne-Hückstädt, U., Schwan, W., Kraus, G.: Physical workload at sewing workplaces - an ergonomic intervention study. In: Pikaar, R.N., Koningsveld, E.A.P., Settels, P.J.M. (eds.) Proceedings IEA 2006, Elsevier Ltd., Oxford (2006)

11. Ditchen, D., Ellegast, R.P., Herda, C., Hoehne-Hückstädt, U.: Ergonomic intervention on musculoskeletal discomfort among crane operators at waste-to-energy-plants. In: Bust, P.D., McCabe, P.T. (eds.) Contemporary Ergonomics 2005, pp. 22-26. Taylor \& Francis, London (2005)

12. Glitsch, U., Hermanns, I., Hamburger, R., Ellegast, R.P., Schüler, R.: EMG-SignalProzessor-Modul zur Langzeitanalyse. In: Grieshaber, R., Stadeler, M., Scholle, H.-C. (eds.) Prävention von arbeitsbedingten Gesundheitsgefahren und Erkrankungen, pp. 501507, Verlag Dr. Bussert \& Stadeler, Jena (2005)

13. Glitsch, U., Keller, S., Kusserow, H., Hermanns, I., Ellegast, R.P., Hüdepohl, J.: Physical and physiological workload profiles of overhead line service technicians. In: Pikaar, R.N., Koningsveld, E.A.P., Settels, P.J.M (eds.) Proceedings IEA 2006, Elsevier Ltd., Oxford (2006) 